\title{
Diferencias en razonamiento analógico y procesos del lenguaje oral en estudiantes de primaria bilingües: quechua-castellano, shipibo - castellano e hispano hablantes
}

\author{
Differences in analogical reasoning and oral language processes in \\ bilingual primary students: quechua-castilian, shipibo-castilian and \\ spanish speakers
}

\author{
Esther Velarde Consoli ${ }^{1}$ \\ Ricardo Canales Gabriel ${ }^{2}$ \\ Carmen Magali Meléndez Jara ${ }^{3}$ \\ Katherine Susana Lingán Huamán ${ }^{4}$ \\ Universidad Nacional Mayor de San Marcos
}

Recibido: $26-11-18$

Aceptado: $27-12-18$

\begin{abstract}
Resumen
La investigación tuvo como objetivo establecer si existen diferencias en el razonamiento analógico y los procesos del lenguaje oral en niños de escuelas con educación en castellano con influencia de lenguas originarias frente a niños hispanohablantes. Se evaluaron 24 alumnos de Socos, 30 de Huamanga, 30 de la comunidad Shipiba y 30 alumnos hispanohablantes de Pachacútec. Se encontraron diferencias significativas en aspectos como la discriminación de fonemas, fono articulatorio, memoria verbal, composición de frases, descripción de acciones, Vocabulario, inteligencia no verbal, a favor de los alumnos de Pachacútec- Ventanilla (los de menor interferencia lingüística), seguidos de los alumnos de Huamanga-Ayacucho; siendo los estudiantes de la comunidad campesina de Socos-Ayacucho. Lo mismo ocurrió cuando se comparó el rendimiento en razonamiento analógico.

Se confirma que exponer a los hablantes de lenguas originarias a una educación en español no permite construir adecuadamente su estructura psicolingüística ni su pensamiento analógico. Palabras clave: Bilingüismo; lenguaje oral; inteligencia.
\end{abstract}

1 Universidad Nacional Mayor de San Marcos, Facultad de Psicología. Lima, Perú. Docente asociada e investigadora. E-mail: emvelarde@hotmail.com

2 Universidad Nacional Mayor de San Marcos, Facultad de Psicología. Lima, Perú. Docente asociado e investigador. E-mail: rcanalesg@unmsm.edu.pe

3 Universidad Nacional Mayor de San Marcos, Facultad de Psicología. Lima, Perú. Docente auxiliar e investigadora. E-mail: carmen75docencia@gmail.com

4 Docente de la Universidad "San Ignacio de Loyola”. E-mail: ksusanalingan39@gmail.com

(C) Los autores. Este artículo es publicado por la Revista de Investigación en Psicología de la Facultad de Psicología, Universidad Nacional Mayor de San Marcos. Este es un artículo de acceso abierto, distribuido bajo los términos de la licencia Creative Commons Atribucion - No Comercia_Compartir Igual 4.0 Internacional. (http://creativecommons.org/licenses/by-nc-sa/4.0/) que permite el uso no comercial, distribución y reproducción en cualquier medio, siempre que la obra original sea debidamente citada. 


\begin{abstract}
The objective of the present investigation was to establish whether there are differences in analogical reasoning and oral language processes in children from schools with education in Spanish with the influence of native languages versus Spanish speakers. 24 Socos students were evaluated; 30 from Huamanga, 30 from the Shipiba community and 30 Spanish speakers from Pachacútec. Significant differences were found in aspects such as phoneme discrimination, articulatory phonology, verbal memory, composition of sentences, description of actions, vocabulary, non-verbal intelligence, in favor of Pachacútec-Ventanilla students (those with the least linguistic interference), followed by students from Huamanga-Ayacucho; being the students of the rural community of Socos-Ayacucho. The same happened when the performance in non-verbal intelligence was compared. It is confirmed that exposing speakers of native languages to an education in Spanish it does not allow to properly construct its psycholinguistic structure or its analogical thinking.
\end{abstract}

Keywords: Bilingualism; oral language; intelligence.

\title{
INTRODUCCIÓN
}

Algunos investigadores (De Houwer, 1999; Deshays, 1990; Duverger, 1995; Siguan, 1996 y Tunmer, Pratt y Herriman, 1984) consideran que el bilingüismo constituye una ventaja cognitiva, puesto que contribuye significativamente a la mejora de sus competencias y habilidades intelectuales. Esta viene a ser una de las conclusiones principales de Signoret (2003), quien luego de revisar teorías e investigaciones sobre el tema, señala que el bilingüismo favorece el desarrollo cognitivo de las personas y el avance socio político. Diversos autores argumentan desde distintas disciplinas como la psicología, la neuropsicología y la psicolingüística que el bilingüismo posee un carácter positivo en tanto favorece el desarrollo de la mente humana. Godijns (1996) sostiene que el bilingüismo promueve no solo el desarrollo de habilidades cognitivas sino también una mayor conciencia metalingüística.

Sin embargo, esta situación no se presenta de la misma manera en todas las realidades puesto que son las condiciones socioculturales que rodean al niño las que determina el tipo y carácter del bilingüismo que se desarrollará. En nuestro país, la población indígena al 2007 estaba constituida por 4'045,713 personas, de las cuales el $83 \%$ son quechuas, $11 \%$ aimaras y un $6 \%$ representan a los pueblos indígenas amazónicos. Actualmente en el Perú se manejan 47 códigos lingüísticos diferentes, y las mayoritarias son el quechua, el aymara en las zonas andinas, y el asháninka y shipibo en la selva (Ministerio de Educación, 2017).

Canales, Velarde, Meléndez y Lingán (2015), en un estudio sobre variaciones dialectales del castellano, utilizando el Test Breve de Bilingüismo (Gonzalez 20016) y una Batería psicolingüística para examinar diversos aspectos del lenguaje oral en estudiantes universitarios en diversas zonas del país, encontraron diferencias en casi todos los aspectos del lenguaje examinado a favor del grupo de estudiantes de Lima. En contraposición, fueron los alumnos de 
Huancavelica (interlecto quechua - castellano), los que mostraron las puntuaciones más bajas, evidenciando serias dificultades para procesar la información vertida en castellano. Igualmente, Canales, Velarde, Meléndez \& Lingán (2014) en una investigación sobre lenguaje oral y habilidades prelectoras en estudiantes de educación inicial de Lima y Vitarte y de zonas rurales (monolingües castellano en Cajamarca y bilingües en Huancavelica) encontraron que la condición de bilingüismo afecta el desarrollo lingüístico y las habilidades pre lectoras en niños de 4 a 6 años de edad.

La condición bilingüe de amplios sectores del país no constituiría un problema si es que existiera en dichas personas un dominio satisfactorio en el uso y manejo de las dos lenguas. Esto es dominio de reglas fonológicas, morfosintácticas, léxicas, semánticas y pragmáticas de cada uno de los idiomas. Sólo en estos casos la condición bilingüe enriquecería al sujeto cognitivamente. Esto es lo que se denomina bilingüismo aditivo. Lamentablemente esto no se da en nuestro país. Muy por el contrario, nuestros estudiantes de lenguas originarias son sometidos a una segunda lengua, en este caso al castellano, en tanto idioma oficial, y ello se prolonga en la instrucción escolar (y en ello, la enseñanza de la lectoescritura), así como en la vida social. A esta condición se le denomina bilingüismo sustractivo (González, 2006).

De otro lado en relación a los aspectos cognitivos, es importante destacar, que en un plano general Bernstein (1966) planteó que en el desarrollo de la inteligencia general, la presencia del razonamiento analógico cumple un rol fundamental, lo cual puede evidenciarse con claridad a partir de los diez años.

En nuestro medio, Abregú $(2008,2009)$ en sus estudios sobre evolución del razonamiento analógico en niños de seis a once años en el Perú, concluye en que dicha habilidad se halla presente en los niños a más temprana edad de lo que consideraría el enfoque piagetano, coincidiendo mas bien con los enfoques de Inguanzo y De la Uz (1996) y Rodríguez-Mena (1993) quienes sostienen que las diferencias individuales obedecerían más bien a factores sociales y culturales.

Considerando que al interior de las funciones ejecutivas, se toma en cuenta, el planeamiento, la flexibilidad mental, la capacidad de abstracción y también el razonamiento, algunas investigaciones señalan la asociación entre condición socio-económica y las funciones ejecutivas (Arán, 2011).

Tomando en cuentas estas consideraciones, el presente estudio tuvo como Objetivo, encontrar las posibles diferencias en los subsistemas del lenguaje oral y el razonamiento analógico en estudiantes de escuelas donde se imparte educación en lengua castellana pero con presencia básica del quechua y/o lenguas amazónicas 
(shipibo), en comparación con estudiantes que no presentaban interferencias lingüísticas de ese tipo (niños hispanohablantes).

En tal sentido, la investigación se planteó el siguiente problema científico:

¿Existen diferencias en el razonamiento analógico y los procesos del lenguaje oral entre los estudiantes de primer y segundo grado de primaria en condición bilingüe quechua-castellana, amazónica- castellano en comparación con los monolingües castellano?

\section{METODO}

El presente estudio corresponde a una Investigación Sustantiva Descriptiva pues se orientó a identificar los niveles de desarrollo del razonamiento analógico y los sub procesos de lenguaje oral en estudiantes de escuelas donde se imparte educación en lengua castellana pero que tienen como base mental y lingüística, lenguas originarias como el quechua y/o las lenguas amazónicas. En cuanto al diseño se utilizó un Diseño descriptivo comparativo debido a que se establecieron diferencias en los rendimientos en los distintos componentes del lenguaje oral y en inteligencia no verbal (razonamiento analógico).

\section{Población y muestra}

La población accesible (Sánchez y Reyes, 2008) estuvo constituida por los estudiantes de primer y segundo grado del nivel socio- económico bajo de una zona rural en Ayacucho: Socos, que reciben enseñanza de carácter bilingüe quechuacastellano; así como por niños de primer y segundo grado de una escuela pública de Huamanga, con menor influencia bilingüe. Y por el otro lado, por alumnos del nivel socio- económico bajo, de la capital: los niños de primer y segundo grado de la Escuela intercultural bilingüe shipibo- castellano en el Rímac, Cantagallo. Todos ellos comparados con niños hispano hablantes de primer y segundo grado de la zona de Pachacútec en Ventanilla, Callao.

De acuerdo a Hernández, Fernández y Baptista (2014) el diseño de la muestra fue no probabilístico de tipo intencionado Se tomó como sub muestras a 24 alumnos de escuelas intercultural bilingüe de la zona rural de Socos, Ayacucho que recibían educación en castellano pero en cuyo entorno existía un alto nivel de influencia quechua; 30 alumnos de Huamanga en Ayacucho con enseñanza predominante en español, con menor grado de influencia quecha, 30 alumnos de la comunidad Shipiba ubicados en Cantagallo en Lima, a quienes también se les impartía educación en castellano y por último, 30 alumnos hispanohablantes de Pachacútec, en el distrito Ventanilla de la región del Callao. A continuación, presentamos los datos. 
Tabla 1

Característica de la muestra

\begin{tabular}{lcccc}
\hline & Socos & Huamanga & Cantagallo & Ventanilla \\
\hline $\mathbf{N}$ & 24 & 30 & 30 & 30 \\
\hline Género & & & & \\
$\quad$ Masculino & $10(41.7 \%)$ & $14(46.7 \%)$ & $20(66.7 \%)$ & $10(33.3 \%)$ \\
$\quad \begin{array}{l}\text { Femenino } \\
\text { Grado escolar }\end{array}$ & $14(58.3 \%)$ & $16(53.3 \%)$ & $10(33.3 \%)$ & $20(66.7 \%)$ \\
$\quad$ ler. grado & $13(54.2 \%)$ & $13(43.3 \%)$ & $16(53.3 \%)$ & $15(50 \%)$ \\
2do. grado & $11(45.8 \%)$ & $17(56.7 \%)$ & $14(46.7 \%)$ & $15(50 \%)$ \\
Lengua materna & & & & \\
$\quad \begin{array}{l}\text { Castellano } \\
\text { Quechua }\end{array}$ & $1(4.2 \%)$ & $16(53.3 \%)$ & $16(53.3 \%)$ & $30(100 \%)$ \\
$\quad \begin{array}{l}\text { Shipibo } \\
\text { Castellano y quechua }\end{array}$ & $18(75 \%)$ & $14(46.7 \%)$ & $1(3.3 \%)$ & - \\
$\quad$ Castellano y shipibo & $5(20.8 \%)$ & - & - & - \\
\hline
\end{tabular}

\section{Técnicas e instrumentos de recolección de datos}

Se utilizaron la prueba Evaluación del Lenguaje Oral (ELO) de Ramos, Fernández y Cuadrado (2008), adaptada por Meléndez M, (2015) y el Test de Matrices Progresivas para evaluar funciones intelectuales de J. C. Raven (2008).

La Prueba de Evaluación del Lenguaje Oral (ELO), cuenta con los subtest de Discriminación Auditiva de Fonemas que evalúa la capacidad de distinguir si dos palabras son iguales o diferentes; el subtest de Aspectos Fonológicos que mide la articulación correcta de palabras con diferente estructuración lingüística; en la parte sintáctica cuenta con la Memoria Verbal de Frases que evalúa la capacidad de retener palabras conformantes de una oración; el subtest de Composición Oral de Frases que solicita al examinado que invente una oración dada una palabra; el subtest de Descripción de Acciones donde el examinado deberá construir una oración, sintácticamente adecuada, que describa dos láminas y, en la parte semántica, cuenta con el subtest de Vocabulario I donde el niño debe señalar el dibujo que corresponde a la definición emitida por el examinador, Vocabulario II donde el sujeto deberá expresar el significado de una palabra dada y el subtest de Comprensión Oral de Narraciones donde el niño, luego de escuchar una historia debe responder preguntas de comprensión oral.

La adaptación del ELO fue realizada por Meléndez (2015) alcanzando, a través de cinco jueces expertos, una $\mathrm{V}$ de Aiken de 1.00 y concordancia entre validez y pertinencia del instrumento. En cuanto a la confiabilidad, el aspecto fonológico alcanzó un alfa 0.90 ; el sintáctico de 0.50 y el semántico de 0.85 . 
El test de Raven, por su parte, mide la inteligencia no verbal libre de influencia cultural y lingüística; particularmente evalúa el razonamiento de tipo analógico. Las personas deben identificar las piezas faltantes de una matriz expuesta en láminas, poniendo en funcionamiento las habilidades perceptuales pero fundamentalmente aplicando reglas de razonamiento analógico y abstracto. Es de administración individual o colectiva. Sobre su validez y confiabilidad (Raven, 2008), se estableció su confiabilidad, obteniendo cifras que oscilan de 0.87 a 0.81 En validez, según el criterio de Terman Merrill, se obtuvo un índice de 0.86 .

Delgado (2002) realiza un análisis psicométrico del Raven Escala especial con 2,496 alumnos del $1^{\circ}$ al $6^{\circ}$ de primaria de colegios de Lima Metropolitana. A nivel de Confiabilidad total medido por las correlaciones ítems- test, se halló un valor de coeficiente Kuder Richardson de 0,91 (alta confiabilidad). Sobre la Validez, aplicando el método de la factorización de los componentes, se halló que un solo factor explica el $76 \%$ de la varianza de las puntuaciones.

\section{RESULTADOS}

En primer término, se trató de establecer si existía algún grado de asociación entre los distintos componentes del lenguaje oral: sub sistemas fonoarticulatorios, léxicos, semánticos y sintácticos, medidos a través de las tareas de discriminación de fonemas, aspectos fonológicos, memoria verbal, composición de oraciones, describir acciones, vocabulario y comprensión oral; con el nivel de inteligencia no verbal medida a través del razonamiento analógico.

\section{Correlaciones entre el lenguaje oral y razonamiento analógico}

Tabla 2

Correlaciones entre las escalas del lenguaje oral y razonamiento analógico

\begin{tabular}{|c|c|c|c|c|c|c|c|c|}
\hline & 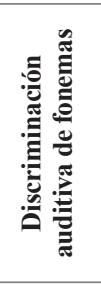 & 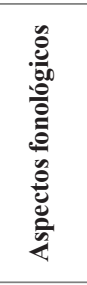 & 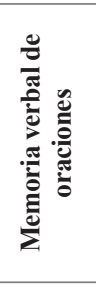 & 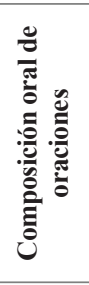 & 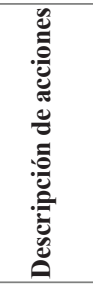 & 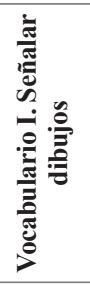 & 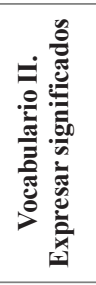 & 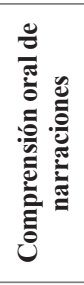 \\
\hline Escala A & .056 & .176 & $.404 * *$ & $.275^{* *}$ & $.324 * *$ & $.216^{*}$ & $.328^{* *}$ & $.206^{*}$ \\
\hline Escala Ab & $.278^{* *}$ & $.237^{*}$ & $.425 * *$ & $.451 \% *$ & $.255^{* *}$ & $.258^{* *}$ & $.330 * *$ & $.365^{* *}$ \\
\hline Escala B & $.306^{* *}$ & $.362 * *$ & $.497 * *$ & $.483 * *$ & $.212^{*}$ & $.255^{* *}$ & $.387 * *$ & $.288^{* *}$ \\
\hline Raven & $.279 * *$ & $.316^{* *}$ & $.536 * *$ & $.498 * *$ & $.328 * *$ & $.287 * *$ & $.432 * *$ & $.358^{* *}$ \\
\hline
\end{tabular}

Nota: coeficientes rho de Spearman; $\mathrm{N}=114$.

$* \mathrm{p}<.05 ; * * \mathrm{p}<.01$ 
Observando la tabla 2, luego de aplicar los estadísticos correspondientes, se aprecia relación significativa entre la mayoría de pares de escalas. Sin embargo, se destacan las correlaciones moderadas entre la tarea de memoria verbal de oraciones y las escalas del test de Raven, así como, las correlaciones entre la tarea de composición oral de oraciones y las escalas Ab, B y la escala total del test de Raven.

En cuanto al rendimiento obtenido en cada uno de los componentes del lenguaje oral en las cuatro realidades socio lingüísticas se encontró lo siguiente:

\section{Diferencias en función de la condición lingüística en el desempeño en Lenguaje oral}

Tabla 3

Resultados alcanzados por los grupos en las tareas de la dimensión fono-articulatoria del lenguaje oral

\begin{tabular}{lccccccc}
\hline & \multicolumn{5}{c}{ Rango promedio } & \multirow{2}{*}{$\mathbf{X}^{2 \mathbf{a}}$} & $\mathbf{p}^{\mathbf{a}}$ \\
\cline { 2 - 6 } & Socos & Huamanga & Cantagallo & Ventanilla & & \\
\hline Discriminación auditiva de fonemas & 65.25 & 48.53 & 49.08 & 68,68 & 10.123 & $\mathbf{. 0 1 8}$ \\
Sílabas directas & 45.10 & 62.77 & 59.03 & 60.62 & 11.169 & $\mathbf{. 0 1 1}$ \\
Sílabas inversas y mixtas & 50.98 & 59.30 & 59.30 & 59.12 & 4.516 & .211 \\
Sílabas complejas -r- & 60.42 & 61.00 & 47.25 & 61.92 & 7.591 & .055 \\
Sílabas complejas -l- & 60.81 & 61.87 & 48.45 & 59.53 & 7.142 & .068 \\
Palabras largas con sílabas complejas & 60.69 & 53.28 & 48.02 & 68.65 & 6.789 & .079 \\
Aspectos fonológicos & 59.08 & 57.48 & 46.75 & 67.00 & 5.815 & .121 \\
\hline
\end{tabular}

a Prueba de Kruskal Wallis (3 grados de libertad)

Se hallaron diferencias significativas entre los alumnos de los distintos grupos estudiados en las tareas de discriminación auditiva de fonemas y en la articulación de palabras con sílabas directas.

Haciendo un análisis complementario de pares (Tabla 4) se observa que las diferencias en la discriminación de fonemas se dan entre los alumnos de Huamanga y los de Ventanilla $(\mathrm{U}=285.500, \mathrm{p}=.009)$, y entre los de la Comunidad de Cantagallo y los de Ventanilla $(\mathrm{U}=290.000, \mathrm{p}=.011)$, en ambos casos las diferencias se dieron a favor de los estudiantes de Ventanilla. De otro lado, las diferencias en la articulación de palabras con silabas directas se dio entre los estudiantes de Socos y Huamanga $(\mathrm{U}=248.000, \mathrm{p}=.005)$; los alumnos de Socos y de la Comunidad Shipiba $(\mathrm{U}=271.000, \mathrm{p}=.037)$; y los estudiantes de Socos y los de Ventanilla $(U=263.500, p=.020)$. En todos los casos, los estudiantes de Socos obtienen las puntuaciones más bajas. 
Llama la atención que las puntuaciones de los estudiantes de Socos en los aspectos fonoarticulatorios sean adecuadas en casi todas las estructuras lingüísticas con excepción de la tarea de repetición de palabras con sílabas directas, en la que logran los puntajes más bajos, en contraste a sus pares, de las otras realidades socio culturales.

\section{Tabla 4}

Diferencias de pares en Discriminación de fonemas y repetición de palabras

\begin{tabular}{|c|c|c|c|c|c|c|}
\hline Escalas & Grupos & $\mathbf{n}$ & $\begin{array}{c}\text { Rango } \\
\text { promedio }\end{array}$ & $\mathbf{U}$ & $\mathbf{p}$ & $\mathbf{r}_{b}$ \\
\hline \multirow{12}{*}{$\begin{array}{l}\text { Discriminación } \\
\text { auditiva de fonemas }\end{array}$} & Socos & 24 & 31.63 & 261.000 & .067 & .25 \\
\hline & Huamanga & 30 & 24.20 & & & \\
\hline & Socos & 24 & 31.58 & 262.000 & .070 & .25 \\
\hline & Cantagallo & 30 & 24.23 & & & \\
\hline & Socos & 24 & 27.04 & 349.000 & .826 & .03 \\
\hline & Ventanilla & 30 & 27.87 & & & \\
\hline & Huamanga & 30 & 30.32 & 444.500 & .923 & .01 \\
\hline & Cantagallo & 30 & 30.68 & & & \\
\hline & Huamanga & 30 & 25.02 & 285.500 & .009 & .34 \\
\hline & Ventanilla & 30 & 35.98 & & & \\
\hline & Cantagallo & 30 & 25.17 & 290.000 & .011 & .33 \\
\hline & Ventanilla & 30 & 35.87 & & & \\
\hline \multirow[t]{12}{*}{ Sílabas directas } & Socos & 24 & 22.83 & 248.000 & .005 & .38 \\
\hline & Huamanga & 30 & 31.23 & & & \\
\hline & Socos & 24 & 23.79 & 271.000 & .037 & .28 \\
\hline & Cantagallo & 30 & 30.47 & & & \\
\hline & Socos & 24 & 23.48 & 263.500 & .020 & .32 \\
\hline & Ventanilla & 30 & 30.72 & & & \\
\hline & Huamanga & 30 & 31.50 & 420.000 & .393 & .11 \\
\hline & Cantagallo & 30 & 29.50 & & & \\
\hline & Huamanga & 30 & 31.03 & 434.000 & .621 & .06 \\
\hline & Ventanilla & 30 & 29.97 & & & \\
\hline & Cantagallo & 30 & 30.07 & 437.000 & .730 & .05 \\
\hline & Ventanilla & 30 & 30.93 & & & \\
\hline
\end{tabular}


Observando la tabla 5, se aprecian diferencias significativas entre los grupos, en los items de memoria verbal de oraciones, composición de oraciones dada una palabra y descripción de acciones a partir de una lámina.

Tabla 5

Resultados obtenidos en las tareas de memoria verbal como parte de la dimensión sintáctica del lenguaje oral

\begin{tabular}{lccccccc}
\hline & \multicolumn{5}{c}{ Rango promedio } & \multirow{2}{*}{$\mathbf{X}^{2 \mathbf{a}}$} & \multirow{2}{*}{$\mathbf{p}^{\mathbf{a}}$} \\
\cline { 2 - 5 } & Socos & Huamanga & Cantagallo & Ventanilla & & \\
\hline Memoria verbal de oraciones & 31.63 & 57.85 & 55.22 & 80.13 & 29.302 & $\mathbf{. 0 0 0}$ \\
Composición de oraciones & 35.67 & 60.78 & 60.07 & 69.12 & 14.909 & $\mathbf{. 0 0 2}$ \\
Descripción de acciones & 26.25 & 51.90 & 74.57 & 71.03 & 35.794 & .000 \\
\hline
\end{tabular}

a Prueba de Kruskal-Wallis (grados de libertad: 3)

Complementariamente cuando se hace el análisis de comparación de pares (Tabla 6) con el estadístico de Mann Whitney se halló que las diferencias en la memoria verbal de oraciones se dan entre los alumnos de Socos y los de Huamanga $(\mathrm{U}=200.000, \mathrm{p}=.005)$, los alumnos de Socos y los de la Comunidad de Cantagallo $(\mathrm{U}=196.000, \mathrm{p}=.004)$, y los estudiantes de Socos y los de Ventanilla ( $\mathrm{U}$ $=63.000, \mathrm{p}=.000)$; en todos los casos, los estudiantes de Socos logran los puntajes más bajos. También, se encontraron diferencias entre los niños de Huamanga y los de Ventanilla $(\mathrm{U}=278.500, \mathrm{p}=.011)$ y los niños de la Comunidad Cantagallo y los de Ventanilla $(U=239.500, p=.002)$, en ambos casos, son los estudiantes de Ventanilla los que obtienen mayores puntajes.

De otro lado, las diferencias en la composición de oraciones dada una palabra se dan entre los alumnos de Socos y Huamanga $(U=188.000, p=.002)$; los alumnos de Socos y los de la Comunidad Shipiba $(U=215.500, p=.011)$; y los alumnos de Socos y los de Ventanilla $(U=152.500, p=.000)$. En todos los casos, los estudiantes de Socos obtienen las puntuaciones más bajas.

Por último, en cuanto a las diferencias en la descripción de acciones a partir de una lámina, éstas se dan entre los alumnos de Socos y Huamanga $(\mathrm{U}=$ $172.500, \mathrm{p}=.001)$; los niños de Socos y los de la Comunidad Shipiba $(\mathrm{U}=70.500$, $\mathrm{p}=.000)$; y los alumnos de Socos y los de Ventanilla $(\mathrm{U}=87.000, \mathrm{p}=.000)$. Los niños de Socos obtienen las puntuaciones más bajas. Además, se encontraron diferencias entre los niños de Huamanga y los de la comunidad de Cantagallo (U $=255.500 ; \mathrm{p}=.004), \mathrm{y}$ los niños de Huamanga y los de Ventanilla $(\mathrm{U}=289.000, \mathrm{p}=$ .016); en los casos vistos, los niños de Huamanga son quienes obtienen los puntajes más bajos. 
Tabla 6

Diferencias de pares en Dimensión sintáctica

\begin{tabular}{|c|c|c|c|c|c|c|}
\hline Escalas & Grupos & n & Rango promedio & $\mathbf{U}$ & $\mathbf{p}$ & $\mathbf{r}_{\mathrm{b}}$ \\
\hline \multirow{12}{*}{$\begin{array}{l}\text { Memoria verbal de } \\
\text { oraciones }\end{array}$} & Socos & 24 & 20.83 & 200.000 & .005 & .38 \\
\hline & Huamanga & 30 & 32.83 & & & \\
\hline & Socos & 24 & 20.67 & 196.000 & .004 & .39 \\
\hline & Cantagallo & 30 & 32.97 & & & \\
\hline & Socos & 24 & 15.13 & 63.000 & .000 & .70 \\
\hline & Ventanilla & 30 & 37.40 & & & \\
\hline & Huamanga & 30 & 31.23 & 428.000 & .743 & .004 \\
\hline & Cantagallo & 30 & 29.77 & & & \\
\hline & Huamanga & 30 & 24.78 & 278.500 & .011 & .33 \\
\hline & Ventanilla & 30 & 36.22 & & & \\
\hline & Cantagallo & 30 & 23.48 & 239.500 & .002 & .41 \\
\hline & Ventanilla & 30 & 37.52 & & & \\
\hline \multirow[t]{12}{*}{ Composición de oraciones } & Socos & 24 & 20.33 & 188.000 & .002 & .41 \\
\hline & Huamanga & 30 & 33.23 & & & \\
\hline & Socos & 24 & 21.48 & 215.500 & .011 & .35 \\
\hline & Cantagallo & 30 & 32.32 & & & \\
\hline & Socos & 24 & 18.85 & 152.500 & .000 & .50 \\
\hline & Ventanilla & 30 & 34.42 & & & \\
\hline & Huamanga & 30 & 30.45 & 448.500 & .982 & .00 \\
\hline & Cantagallo & 30 & 30.55 & & & \\
\hline & Huamanga & 30 & 28.10 & 378.000 & .282 & .14 \\
\hline & Ventanilla & 30 & 32.90 & & & \\
\hline & Cantagallo & 30 & 28.20 & 381.000 & .305 & .13 \\
\hline & Ventanilla & 30 & 32.80 & & & \\
\hline \multirow[t]{12}{*}{ Descripción de acciones } & Socos & 24 & 19.69 & 172.500 & .001 & .45 \\
\hline & Huamanga & 30 & 33.75 & & & \\
\hline & Socos & 24 & 15.44 & 70.500 & .000 & .69 \\
\hline & Cantagallo & 30 & 37.15 & & & \\
\hline & Socos & 24 & 16.13 & 87.000 & .000 & .65 \\
\hline & Ventanilla & 30 & 36.60 & & & \\
\hline & Huamanga & 30 & 24.02 & 255.500 & .004 & .37 \\
\hline & Cantagallo & 30 & 36.98 & & & \\
\hline & Huamanga & 30 & 25.13 & 289.000 & .016 & .31 \\
\hline & Ventanilla & 30 & 35.87 & & & \\
\hline & Cantagallo & 30 & 31.43 & 422.000 & .675 & .05 \\
\hline & Ventanilla & 30 & 29.57 & & & \\
\hline
\end{tabular}


Tabla 7

Resultados de los grupos en las tareas de la dimensión semántica del lenguaje oral

\begin{tabular}{lccccccc}
\hline & \multicolumn{5}{c}{ Rango promedio } & \multirow{2}{*}{$\mathbf{X}^{2 \mathbf{a}}$} & $\mathbf{p}^{\mathbf{a}}$ \\
\cline { 2 - 5 } & $\mathbf{S o c o s}$ & Huamanga & Cantagallo & Ventanilla & & \\
\hline Vocabulario I. Señalar dibujos & 42.00 & 51.15 & 61.18 & 72,57 & 14.169 & $\mathbf{. 0 0 3}$ \\
Vocabulario II. Expresar significados & 36.63 & 46.63 & 69.80 & 72.77 & 23.909 & $\mathbf{. 0 0 0}$ \\
Comprensión oral de narraciones & 56.46 & 47.05 & 58.02 & 68.27 & 6.429 & .092 \\
\hline
\end{tabular}

a Test de Kruskal-Wallis (grados de libertad: 3)

Se aprecia en la tabla 7 que existen diferencias significativas en el rendimiento de los alumnos de las distintas realidades sociales y lingüísticas en las tareas de vocabulario I (señalar dibujos al oír su significado funcional) y vocabulario II (expresar significados de las palabras mencionadas).

Igualmente de modo complementario, cuando se hace el análisis por pares (Tabla 8) de acuerdo al estadístico de Mann Whitney, se encontró que las diferencias en la escala de vocabulario I se presentan entre: los alumnos de Socos y los de la Comunidad de Cantagallo ( $\mathrm{U}=238.500, \mathrm{p}=.027)$, y los estudiantes de Socos y los de Ventanilla $(\mathrm{U}=179.001, \mathrm{p}=.000)$; en ambos los casos, los alumnos de Socos obtienen las puntuaciones más bajas. También, se encontraron diferencias entre los niños de Huamanga y los de Ventanilla $(\mathrm{U}=273.500, \mathrm{p}=.006)$, y los niños de la Comunidad Cantagallo y los de Ventanilla $(\mathrm{U}=239.500, \mathrm{p}=.002)$, en ambos casos, son los estudiantes de Ventanilla los que obtienen mayores puntajes.

De otro lado, en cuanto a las diferencias en la escala de vocabulario II se dan entre los alumnos de Socos y los de la Comunidad de Cantagallo ( $U=165.500$, $\mathrm{p}=.001)$; y los niños de Socos y los de Ventanilla $(\mathrm{U}=123.000, \mathrm{p}=.000)$; en ambos casos, los estudiantes de Socos obtienen las puntuaciones más bajas. Además, también se encontraron diferencias entre las puntuaciones de los niños de Huamanga y los de la comunidad de Cantagallo ( $\mathrm{U}=275.500, \mathrm{p}=.009)$; y los niños de Huamanga y los de Ventanilla $(\mathrm{U}=229.000, \mathrm{p}=.001)$; en los dos casos, son los estudiantes de Huamanga los que obtienen las menores puntuaciones.

En cuanto a la comprensión oral de narraciones no se observaron diferencias entre los grupos evaluados.

De acuerdo a la tabla 9 se observa que hay diferencias significativas en los alumnos de diferentes realidades en su rendimiento en las escalas y en la puntuación global del Test de Raven para el razonamiento analógico.

Haciendo el análisis respectivo de pares con el Mann Whitney (Tabla 10) se halló que las diferencias se presentan entre los niños de Socos y cada uno de 
los otros grupos de alumnos (Huamanga: $\mathrm{U}=159.500, \mathrm{p}=.000$; Cantagallo: $\mathrm{U}$ $=111.000, p=.000$; Ventanilla: $U=61.000, p=.000$ ). Nuevamente, los alumnos de Socos obtienen puntajes inferiores. También, se encontraron diferencias en la escala $\mathrm{Ab}$ entre los niños de Huamanga y los de Ventanilla $(\mathrm{U}=277.500, \mathrm{p}=.010)$, y los niños de la Comunidad Cantagallo y los de Ventanilla $(U=288.000, p=.015)$, en la misma escala. En ambos casos, son los estudiantes de Ventanilla los que obtienen mayores puntajes.

\section{Tabla 8}

Diferencias de pares en Dimensión semántica

\begin{tabular}{|c|c|c|c|c|c|c|}
\hline Escalas & Grupos & n & Rango promedio & $\mathbf{U}$ & $\mathbf{p}$ & $\mathbf{r}_{\mathrm{b}}$ \\
\hline \multirow{12}{*}{$\begin{array}{l}\text { Vocabulario I. } \\
\text { Señalar dibujos }\end{array}$} & Socos & 24 & 24.60 & 290.500 & .194 & .18 \\
\hline & Huamanga & 30 & 29.82 & & & \\
\hline & Socos & 24 & 22.44 & 238.500 & .027 & .30 \\
\hline & Cantagallo & 30 & 31.55 & & & \\
\hline & Socos & 24 & 19.96 & 179.000 & .001 & .44 \\
\hline & Ventanilla & 30 & 33.53 & & & \\
\hline & Huamanga & 30 & 27.72 & 366.500 & .192 & .17 \\
\hline & Cantagallo & 30 & 33.28 & & & \\
\hline & Huamanga & 30 & 24.62 & 273.500 & .006 & .35 \\
\hline & Ventanilla & 30 & 36.38 & & & \\
\hline & Cantagallo & 30 & 27.35 & 355.500 & .141 & .19 \\
\hline & Ventanilla & 30 & 33.65 & & & \\
\hline \multirow{12}{*}{$\begin{array}{l}\text { Vocabulario } \\
\text { II. Expresar } \\
\text { significados }\end{array}$} & Socos & 24 & 24.60 & 290.500 & .219 & .17 \\
\hline & Huamanga & 30 & 29.82 & & & \\
\hline & Socos & 24 & 19.40 & 165.500 & .001 & .47 \\
\hline & Cantagallo & 30 & 33.98 & & & \\
\hline & Socos & 24 & 17.63 & 123.000 & .000 & .57 \\
\hline & Ventanilla & 30 & 35.40 & & & \\
\hline & Huamanga & 30 & 24.68 & 275.500 & .009 & .34 \\
\hline & Cantagallo & 30 & 36.32 & & & \\
\hline & Huamanga & 30 & 23.13 & 229.000 & .001 & .43 \\
\hline & Ventanilla & 30 & 37.87 & & & \\
\hline & Cantagallo & 30 & 30.50 & 450.000 & 1.000 & - \\
\hline & Ventanilla & 30 & 30.50 & & & \\
\hline
\end{tabular}


Diferencias en razonamiento analógico y procesos del lenguaje oral en estudiantes de primaria bilingües: quechua-castellano, shipibo - castellano e hispano hablantes

Tabla 9

Resultados alcanzados en el Razonamiento analógico

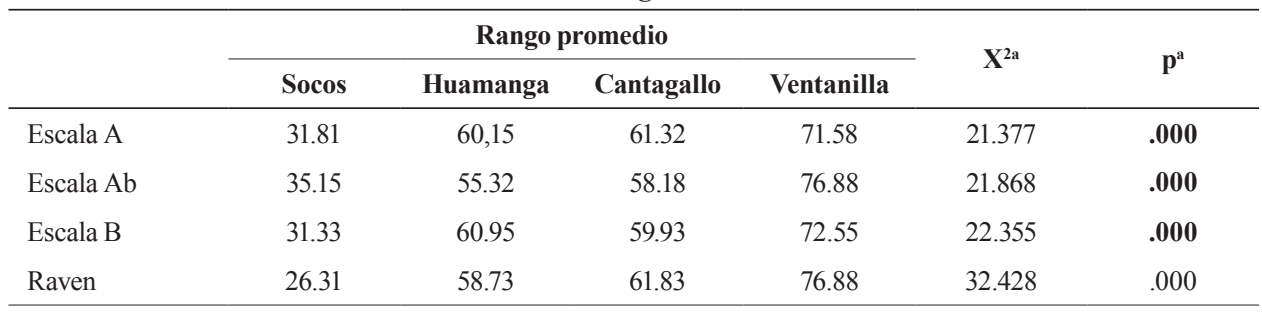

a Test de Kruskal-Wallis (grados de libertad: 3)

Tabla 10

Comparación de grupos según los resultados obtenidos en el Test de Raven (Razonamiento Analógico)

\begin{tabular}{|c|c|c|c|c|c|c|}
\hline Escalas & Grupos & $\mathbf{n}$ & $\begin{array}{c}\text { Rango } \\
\text { promedio }\end{array}$ & $\mathbf{U}$ & $\mathbf{p}$ & $\mathbf{r}_{\mathrm{b}}$ \\
\hline \multirow{12}{*}{ Escala A } & Socos & 24 & 30.31 & 187.500 & .002 & .42 \\
\hline & Huamanga & 30 & 33.25 & & & \\
\hline & Socos & 24 & 19.15 & 159.500 & .000 & .49 \\
\hline & Cantagallo & 30 & 34.18 & & & \\
\hline & Socos & 24 & 17.35 & 116.500 & .000 & .59 \\
\hline & Ventanilla & 30 & 35.62 & & & \\
\hline & Huamanga & 30 & 30.28 & 443.500 & .922 & .13 \\
\hline & Cantagallo & 30 & 30.72 & & & \\
\hline & Huamanga & 30 & 27.62 & 363.500 & .192 & .17 \\
\hline & Ventanilla & 30 & 33.38 & & & \\
\hline & Cantagallo & 30 & 27.42 & 357.500 & .161 & .18 \\
\hline & Ventanilla & 30 & 33.58 & & & \\
\hline \multirow[t]{12}{*}{ Escala Ab } & Socos & 24 & 22.04 & 229.000 & .021 & .31 \\
\hline & Huamanga & 30 & 31.87 & & & \\
\hline & Socos & 24 & 20.90 & 201.500 & .005 & .38 \\
\hline & Cantagallo & 30 & 32.78 & & & \\
\hline & Socos & 24 & 17.21 & 113.000 & .000 & .59 \\
\hline & Ventanilla & 30 & 35.73 & & & \\
\hline & Huamanga & 30 & 29.70 & 426.000 & .720 & .05 \\
\hline & Cantagallo & 30 & 31.30 & & & \\
\hline & Huamanga & 30 & 24.75 & 277.500 & .010 & .33 \\
\hline & Ventanilla & 30 & 36.25 & & & \\
\hline & Cantagallo & 30 & 25.10 & 288.000 & .015 & .31 \\
\hline & Ventanilla & 30 & 35.90 & & & \\
\hline
\end{tabular}




\begin{tabular}{|c|c|c|c|c|c|c|}
\hline Escalas & Grupos & $\mathbf{n}$ & $\begin{array}{c}\text { Rango } \\
\text { promedio }\end{array}$ & $\mathbf{U}$ & $\mathbf{p}$ & $\mathbf{r}_{\mathrm{b}}$ \\
\hline \multirow[t]{12}{*}{ Escala B } & Socos & 24 & 19.73 & 173.500 & .001 & .45 \\
\hline & Huamanga & 30 & 33.72 & & & \\
\hline & Socos & 24 & 19.46 & 167.000 & .001 & .47 \\
\hline & Cantagallo & 30 & 33.93 & & & \\
\hline & Socos & 24 & 17.15 & 111.500 & .000 & .59 \\
\hline & Ventanilla & 30 & 35.78 & & & \\
\hline & Huamanga & 30 & 30.65 & 445.500 & .946 & .01 \\
\hline & Cantagallo & 30 & 30.35 & & & \\
\hline & Huamanga & 30 & 27.58 & 362.500 & .190 & .17 \\
\hline & Ventanilla & 30 & 33.42 & & & \\
\hline & Cantagallo & 30 & 26.65 & 334.500 & .081 & .23 \\
\hline & Ventanilla & 30 & 34.35 & & & \\
\hline \multirow[t]{12}{*}{ Raven } & Socos & 24 & 19.15 & 159.500 & .000 & .48 \\
\hline & Huamanga & 30 & 34.18 & & & \\
\hline & Socos & 24 & 17.13 & 111.000 & .000 & .59 \\
\hline & Cantagallo & 30 & 35.80 & & & \\
\hline & Socos & 24 & 15.04 & 61.000 & .000 & .71 \\
\hline & Ventanilla & 30 & 37.47 & & & \\
\hline & Huamanga & 30 & 29.67 & 425.000 & .711 & .05 \\
\hline & Cantagallo & 30 & 31.33 & & & \\
\hline & Huamanga & 30 & 25.88 & 311.500 & .040 & .27 \\
\hline & Ventanilla & 30 & 35.12 & & & \\
\hline & Cantagallo & 30 & 25.70 & 306.000 & .033 & .28 \\
\hline & Ventanilla & 30 & 35.30 & & & \\
\hline
\end{tabular}

\section{DISCUSIÓN}

En la presente investigación se estudió a niños con niveles moderados y leves de presencia en su estructura lingüística del quechua o de lenguas amazónicas (shipibos) en comparación a estudiantes cuya estructura lingüística básica era el español, y se tuvo como objetivo encontrar cuales eran los grados de dominio de la lengua castellana que viene a ser la lengua oficial en el Perú, y que se aplican en los diversos centros de enseñanza.

Se hallaron diferencias significativas en los niveles de lenguaje oral, tanto en la discriminación auditivas de fonemas, el dominio fonoarticulatorio (menos en sílabas mixtas, complejas y palabras largas) y en las distintas tareas que componen los subtests de aspectos sintácticos y semánticos del lenguaje oral 
(menos en comprensión oral de narraciones), a favor de los alumnos de PachacútecVentanilla (castellano hablantes) los que obtuvieron mejores desempeños en cada una de las tareas, mientras que fueron los estudiantes de Socos (comunidad rural de Ayacucho con presencia considerable del quechua) y en un solo aspecto, los alumnos de Huamanga (discriminación auditiva de fonemas) los que puntuaron más bajo.

Los hallazgos mostrados, refuerzan la importancia de las desventajas socio-culturales señaladas por González (2006): marginación social, pobreza, analfabetismo de los padres, bilingüismo sustractivo, que repercutirían sobre el desarrollo cognitivo en los niños. Ello se daría con mayor preponderancia en las áreas marginales del país en donde predominan la lengua quechua, el aymara o lenguas amazónicas.

Con dichos resultados se evidenciaría que la falta de un tratamiento adecuado de la condición bilingüe de muchos alumnos en el país perjudica la construcción y desarrollo de su estructura lingüística mental. Dicha situación, a su vez, afectará directamente sus procesos de aprendizaje de la lectura, su desempeño escolar, su inserción social y sus perspectivas como ciudadano integrado a la nación peruana.

Esto coincide con lo hallado por Canales, Velarde, Meléndez \& Lingán (2014) quienes hallaron que la condición de bilingüismo afecta el desarrollo lingüístico y las habilidades pre lectoras en niños de 4 a 6 años de edad. Los hallazgos muestran diferencias significativas en el desarrollo del lenguaje oral a favor de los niños de zonas urbanas, en desmedro de los niños de condición rural bilingüe (Quintaojos en Huancavelica) tanto en desarrollo lingüístico oral como en habilidades previas a la lectura.

En cuanto a la inteligencia no verbal también los resultados revelan un mayor desempeño a favor de los estudiantes de Ventanilla (monolingües castellanos), lo cual es preocupante porque estaríamos viendo hasta que punto, dicho problema cultural y lingüístico puede estar afectando el desarrollo de las estructuras cognitivas en el infante.

La línea de estudio sobre bilingüismo y habilidades cognitivas, no reporta mayores antecedentes inmediatos. Fueron, en principio, las investigaciones sobre Formación conceptos en niños bilingües y monolingües en Ayacucho y luego Adquisición del lenguaje en el niño peruano (González, 2006) las referencias más importantes, que luego se han ido complementando con los estudios de Canales, Velarde, Meléndez y Lingán (2014, 2015).

Es interesante señalar que, en nuestro estudio se encontró que las áreas del lenguaje más afectadas fueron las de producción lingüística más que de la comprensión lingüística. Especialmente en tareas relacionadas con la tarea de 
repetir o inventar una oración y de describir acciones (aspectos sintácticos del lenguaje oral) y las de expresar el significado de unas palabras (aspecto semántico). Son operaciones lingüísticas más complejas y de mayor dificultad. En cambio, la tarea de comprender narraciones les resulta más sencilla, posiblemente porque están más familiarizados con estas experiencias tanto en el aula como en la comunidad y la familia.

\section{CONCLUSIONES}

1. En la tarea de discriminación auditiva de fonemas se encontraron diferencias a favor de los estudiantes de Ventanilla siendo los estudiantes de Huamanga los que obtuvieron más bajas puntuaciones.

2. En el subtest fonoarticulatorio se encontraron diferencias sólo en la articulación de sílabas directas siendo los estudiantes de Socos los que obtuvieron las puntuaciones más bajas y los de Huamanga los que lograron puntuaciones más altas. En el resto de articulaciones (de sílabas inversas, mixtas y complejas) no se encontraron diferencias.

3. En los subtests de Procesos Sintácticos (memoria verbal, composición de oraciones y descripción de acciones) se encontraron diferencias en todas las tareas. Aquí los alumnos de Socos obtienen los puntajes más bajos y fueron los estudiantes de Ventanilla (en memoria verbal y composición de oraciones) y Cantagallo (descripción de oraciones) los que obtuvieron mayores puntajes.

4. Encuanto a los Procesos Semánticos se encontraron diferencias significativas en tareas de vocabulario I (señalar dibujos de acuerdo al significado) y Vocabulario II (expresar conceptos), siendo los alumnos de Socos aquellos que obtuvieron los puntajes más bajos y los estudiantes de Ventanilla los que obtuvieron los mayores puntajes. En la tarea de comprensión oral de narraciones no se observaron diferencias.

5. Por último, en cuanto a la inteligencia no verbal medida a partir del razonamiento analógico, los alumnos de Socos obtuvieron puntajes inferiores y los estudiantes de Ventanilla los que obtuvieron mayores puntajes. Cabe señalar que cada uno de los componentes del lenguaje oral mantuvo un grado de asociación con la inteligencia no verbal, en especial la tarea de memoria verbal, composición de oraciones y expresión de significados.

\section{NOTA DE RECONOCIMIENTO}

Queremos agradecer a los estudiantes de psicología y educación que colaboraron con el estudio. Estos fueron: de la Facultad de Psicología de la UNMSM, Juan Ramírez, Angélika Pizarro, Jefferson Vargas, Andrés Pereyra, Helen Silva, 
Paloma Izquierdo y de Educación, Yoly Rodríguez, Anthony Trujillo, Dulce Iglesias, Vanessa Jurado y Anais Pablo.

\section{REFERENCIAS}

Abregú, L. (2008). Verificación del Constructo Teórico de Bernstein en la evolución del razonamiento analógico infantil. Estudio longitudinal. REV ACAD PERU SALUD 15(1), 2008

Abregú, L. (2009). Evolución del razonamiento analógico en niños: seguimiento desde los seis hasta los once años de edad. Avances en Psicología Latinoamericana, vol. 27, núm. 1, enero-junio, 2009, pp. 97-110

Bernstein J. (1966). Test de Matrices Progresivas para la medición de la capacidad intelectual. Escala especial. Buenos Aires: Ed. Paidos.

Canales R., Velarde E., Meléndez M., Lingán S (2014) Lenguaje oral y habilidades prelectoras en niños de 4 a 6 años. Un estudio sobre marginalidad y bilingüismo en el Perú. Revista de Investigación en Psicología, Vol.17- N¹- 107-119.

Canales R., Velarde E., Meléndez M., Lingán S. (2015). Variaciones dialectales del castellano y distancias comunicacionales en estudiantes universitarios iniciales de educación. Un estudio sobre habilidades psicolingüísticas y bilingüismo en el Perú. Revista de Investigación en Psicología. Vol. 18- $\mathrm{N}^{\circ}$ 2, 71-81.

Delgado, A. (2002). Estudio psicométrico del test de matrices progresivas de Raven a colores en estudiantes de primaria de Lima Metropolitana. Revista de Investigación en Psicología, Vol.5 No.2.

De Houwer, A. (1995), Bilingual Language Acquisition. En: Fletcher, P. y MacWhinney, B. The handbook of child language. Londres: Blackwell.

Deshays, E. (1990), L'enfant bilingue, París: Robert Laffont.

Duverger, J. (1995) Reperes et enjeux. Revue Internationale d'Éducation, Enseignements bilingues, núm. 7, 29-44.

Godijns, R. (1996). La edad más idónea para aprender un segundo idioma ¿es necesariamente la primera infancia? En: Siguan, M. (2006). La enseñanza precoz de una segunda lengua en la escuela. Barcelona: Horsoni, pp. 171-80.

González, R. (2006). Problemas psicolingüísticos en el Perú. Obras Completas. Volumen I. Lima: Ediciones. N. Reátegui.

Hernández, R.; Fernández, C. y Baptista, P. (2014). Metodología de la Investigación. México: Mc Graw Hill.

Meléndez, C. (2015). Lenguaje oral y procesos psicológicos de la escritura en escolares con y sin intoxicación por plomo en el Callao. Revista de Investigación en Psicología. Vol. 18- $N^{\circ}$ 1- 2015. 63-74

Ministerio de Educación (2017). Hacia una Educación Intercultural Bilingüe de Calidad. Disponible en: http://www.minedu.gob.pe/minedu/archivos/a/002/01-general/2propuestas pedagogicas eib 2013.pdf 
Ramos S., Cuadrado G., Fernández A. (2008). Prueba de Evaluación del Lenguaje oral (ELO). Lima: Ediciones Libro Amigo.

Raven, J. (2008). Test de Matrices Progresivas para la medida de la Capacidad Intelectual. Escala Especial. Buenos Aires: Paidós.

Sánchez, H. y Reyes, C. (2008). Metodología y diseños en la investigación científica. Lima: Edit. Universitaria. Universidad Ricardo Palma.

Signoret, A. 2003. Bilingüismo y cognición: ¿cuándo iniciar el bilingüismo en el aula? Perfiles Educativos, vol. XXV, núm. 102, Instituto de Investigaciones sobre laUniversidad y la Educación, México.

Siguan, M. (coord.) (2006). La enseñanza precoz de una segunda lengua en la escuela. Barcelona: Horsoni.

Tunmer, E., y Myhill, M. (1984), Metalinguistic awereness and bilingualism. En: Tunmer, W., Pratt, C. y Herriman, M. L. (eds.). Metalinguistic awereness in children. Berlín: Springer Verlag, pp. 169-87. 